\title{
Experimental Assessment of the Accuracy of Modulated and Unmodulated Visible Light Positioning
}

\author{
$1^{\text {st }}$ Sander Bastiaens \\ WAVES, INTEC \\ Ghent University/imec \\ Ghent, Belgium \\ Sander.Bastiaens@UGent.be
}

\author{
$2^{\text {nd }}$ Kenneth Deprez \\ WAVES, INTEC \\ Ghent University/imec \\ Ghent, Belgium \\ Kenneth.Deprez@UGent.be
}

\author{
$3^{\text {rd }}$ Luc Martens \\ WAVES, INTEC \\ Ghent University/imec \\ Ghent, Belgium \\ Luc1.Martens@UGent.be
}

\author{
$4^{\text {th }}$ Wout Joseph \\ WAVES, INTEC \\ Ghent University/imec \\ Ghent, Belgium \\ Wout.Joseph@UGent.be
}

\author{
$5^{\text {th }}$ David Plets \\ WAVES, INTEC \\ Ghent University/imec \\ Ghent, Belgium \\ David.Plets@UGent.be
}

\begin{abstract}
To feature a Light-Emitting Diode (LED) illumination infrastructure as a received signal strength (RSS)-based Visible Light Positioning (VLP) system's beacons, each LED's driver currently needs to be retrofitted. As a consequence of the intensity modulation (IM) retrofit, the LEDs' average radiant flux decreases. To remain adherent to the required illuminance levels, additional LED sources are required, which significantly raises the roll-out cost. The goal of unmodulated VLP (uVLP) is to refrain from modulating the LEDs by exploiting an inherent characteristic of the LEDs' light signals, namely the characteristic frequency $(\mathrm{CF})$. This paper shows the aptitude of using the spectral magnitude at the $C F$ to infer accurate positioning in photodiode (PD)-based RSS uVLP. Compared to regular VLP, uVLP displays a limited increase in median and $90^{\text {th }}$ percentile root-mean-square error of $3.52 \mathrm{~cm}(8.72 \mathrm{~cm} \mathrm{vs.} 5.20 \mathrm{~cm})$ and $7.54 \mathrm{~cm}(16.96 \mathrm{~cm}$ vs. $9.42 \mathrm{~cm})$ respectively, during trilateration in a $4 \mathrm{~m}$ by $4 \mathrm{~m}$ lab setup. This shows uVLP's potential as an indoor localisation system.
\end{abstract}

Index Terms-Unmodulated Visible Light Positioning, uVLP, Visible Light Positioning, VLP, Light Signals Of Opportunity, Characteristic Frequency, Received Signal Strength (RSS), LED, Localisation, Positioning

\section{INTRODUCTION}

Visible Light Communication (VLC) is being researched for application in next-generation broadcasting networks. The Internet of Radio Light (IoRL) framework [1], [2], for instance, integrates VLC into a $5 \mathrm{G}$ network for wireless and mobile connectivity in homes, businesses [3], museums [4] and supermarkets [5]. Several (immersive) broadcasting applications suitable for VLC, depend on a form of location-awareness [6], e.g. wireless augmented and virtual reality [7]. Visible Light Positioning (VLP), as a subdomain of VLC, strives to deliver this required, accurate geographic data.
Typical VLP (and thus VLC) systems employ intensity modulated LED lamps that are frequently part of a pre-existing illumination infrastructure. This reuse of the lighting may be categorised as VLP's main economic driver. However, VLPenabling LED lamps not only entails a LED driver retrofit, it also inadvertently decreases the LEDs' average radiant flux. The result is an elevated roll-out cost as more lamps will be needed to meet the stringent illumination requirements.

Unmodulated VLP (uVLP) hopes to cope with these challenges by leaving the LEDs unmodulated and by utilising the LEDs' light signals of opportunity (LSOOP) to infer (accurate) positioning estimates. Two uVLP approaches can be distinguished, depending on whether (1) the total illuminance or irradiance measured at the receiver is used as localisation input, or (2) de LSOOP signals are first separated into individual LED contributions. In [8], Amsters et al. evaluated the performance of the former by running an iterated extended Kalman filter on the total photovoltage and on encoder values to navigate a photodiode (PD)-equipped mobile robot inside a $10 \mathrm{~m} \times 10 \mathrm{~m}$ area with 4 Lambertian LEDs placed in a square. A simulation experiment showed positioning errors exceeding $50 \mathrm{~cm}$, even with multiple PDs. This and other total illuminance methods run into difficulties in the absence of an illuminance gradient, which frequently occurs in practice as a uniform illuminance distribution is strived for. Zhang et al. in [9] demonstrate that the inherent characteristic frequency $(C F)$ of fluorescent lights (FLs) can be exploited to demultiplex the different FLs' contributions. With a smartphone, a $37 \mathrm{~cm}$ accurate positioning in a $23.7 \mathrm{~m}$ by $6.4 \mathrm{~m}$ by $3.1 \mathrm{~m}$ area can be obtained. 
With the illumination market transitioning to LED technology i.e. the solid-state lighting (r)evolution, (u)VLP also turns to LEDs rather than FLs. In this paper, it is shown that although high-power LED deployments differ extensively from their FL counterparts, their constant current drivers induce resonance frequencies as well. Moreover, the identifiable characteristic frequency $(C F)$ can serve as a discriminative feature in PD-based received signal strength (RSS) uVLP. Based on 2 large datasets collected in a $4 \mathrm{~m}$ by $4 \mathrm{~m}$ lab setup, the trilateration-based positioning root-mean-square error (rMSE) of both UVLP and regular VLP is experimentally compared.

\section{System Description}

\section{A. LEDs' Characteristic Frequency}

LED lamps vary both in their bare LED light sources and their LED driver topologies and complexity, depending on the contemplated market segment and application [10]. The constant current driver found in most LED systems induces resonance frequencies that are noticeable in the LED's electrical output signal. The sole or most prominent of the resonance frequencies is dubbed the characteristic frequency $(C F)$. As is the case for fluorescent lights (FLs), a substantial variation in the LEDs' emitted electrical spectrum is present, allowing the discerning of a unique $C F$ per LED, even for LEDs of the same type. Exploiting the set of $C F \mathrm{~s}$, permits the application of the VLP positioning principles without having to modulate the LEDs.

\section{B. (Positioning) Measurement Setup}

This paper considers BXRE-35E2000-C-73 ${ }^{1}$ Chip on board (COB) LEDs. COB LEDs are favoured in VLP as they are exhibiting approximately Lambertian radiating point source behaviour, and hence allow an easy localisation [11]. The COBs' $C F$ s and associated unmodulated VLP positioning performance is recorded in a dedicated $4 \mathrm{~m}$ by $4 \mathrm{~m}$ VLP lab (Fig. 1) that is surrounded by black cloths to minimise reflections [11].

There, $K=4 B X R E$ LEDs are placed in a quasi-square, $2.25 \mathrm{~m}$ above the receiver plane. To ensure a fair comparison between both uVLP and dedicated VLP, the COB LEDs are driven by an identical LED driver, the LTM8005 Demo Board $^{2}$, in DC and $\mathrm{AC}$ regime respectively. In $\mathrm{AC}$ regime, the LTM8005 driver's frequency $f_{c, i}=2^{i-1} f_{0}, \quad\left(f_{0}=\right.$ $0.5 \mathrm{kHz}, \quad i=1 . . K)$ is set via the WiFi-enabled Adafruit Feather M0 WIFI w/ATWINC $1500^{3}$ module in accordance with the square wave modulation scheme of [12].

At the receiving end, the transmitted signals are found encoded in the total photovoltage signal $V_{P D, i}(t)$ generated by the Thorlabs PDA36A $2^{4}$ module (with a transimpedance gain of $\left.1.51 \cdot 10^{3} \mathrm{~V} / \mathrm{A}[11]\right)$. Placed on a slider system, $V_{P D, i}(t)$ is measured on a uniform $2.5 \mathrm{~cm}$ grid, yielding 24649 measure-

\footnotetext{
${ }^{1}$ https://www.bridgelux.com/sites/default/files/resource_media/Bridgelux\%20DS101\% 20Gen\%207\%20V13\%20Array\%20Data\%20Sheet\%2020190930\%20Rev\%20N.pdf

${ }^{2}$ https://www.analog.com/en/products/ltm8005.html\#product-overview

${ }^{3}$ https://www.adafruit.com/product/3010

${ }^{4}$ https://www.thorlabs.com/thorproduct.cfm?partnumber=PDA36A2
}

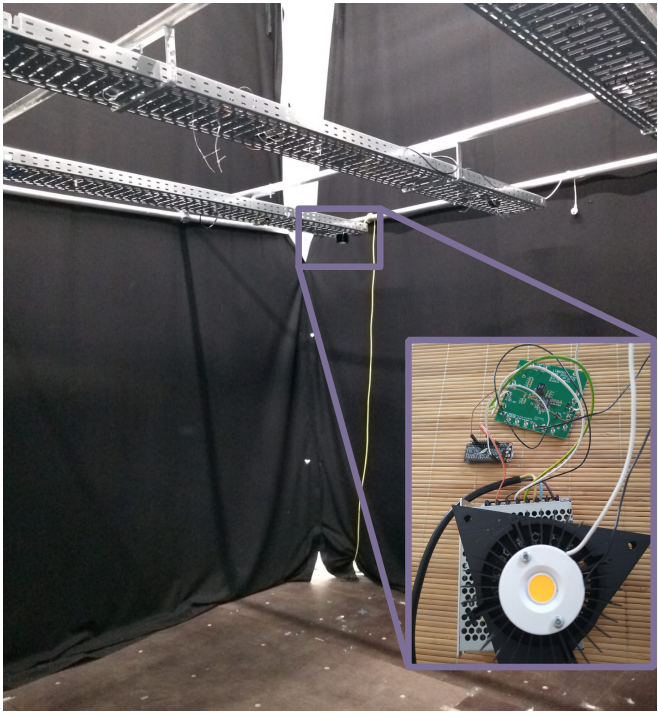

Fig. 1. Illustration of the COB (u)VLP lab environment

ment locations in total. The subsequent receiver chain blocks consist of the National Instrument USB-6212 DAQ device and a MATLAB ${ }^{\circledR}$ backend to both sample $V_{P D, i}(t)$ (during $1 \mathrm{~s}$ at a rate $f_{S}=256 \mathrm{kHz}$ ) and employ it as input for the demodulation and localisation engine.

To determine the LEDs' $C F \mathrm{~s}, V_{P D, i}(t)$ is first measured, with $N=51.2 \mathrm{kS}$ samples, directly underneath each LED whilst the other LEDs are switched off. $100 C F$ measurements are averaged to obtain the final $C F$.

\section{Positioning Approach}

1) Demodulation: From $V_{P D, i}(t)$, the set of the individual LED photovoltage magnitude contributions $\left\{V_{P D, i}\right\}$ at frequencies $\left\{f_{c, i}\right\}$ is demultiplexed via the fast Fourier transform (FFT) amplitude spectrum-based identification method of [12]. $f_{c, i}$ is either the first harmonic frequency in regular VLP [12] or the characteristic frequency in UVLP. To improve demodulation (and positioning) robustness, instead of taking the spectral magnitude found at $f_{c, i}$, a peak detection is opted for. The neighbourhood $2 \Delta f$ of the nominal $f_{c, i}$ i.e. $\left[f_{c, i}-\Delta f, f_{c, i}+\Delta f\right]$ is considered (see Section III-B).

Prior to demodulation, the $V_{P D, i}(t)$ time signal, spanning $1 \mathrm{~s}$, is subdivided in $A V G$ segments to average $\left\{V_{P D, i}\right\}$. Two averaging methods are discerned: (1) the complex FFT spectrum is averaged prior to peak detection of $\left\{V_{P D, i}\right\}$ on the amplitude spectrum, and (2) the peak detected $\left\{V_{P D, i}\right\}$ of each of the segments is averaged. Methods (1) and (2) are respectively denoted by $S P E C T$ (with abbreviation $s$ ) and $P E A K$ (with abbreviation $p$ )

The positioning method of the following Section II-C2 employs the set of per LED received radiant powers $\left\{P_{R, i}\right\}, i=$ $1 . . K$ as RSS values, rather than their photovoltage variants $\left\{V_{P D, i}\right\}$. The individual power contributions $\left\{P_{R, i}\right\}$ are derived from $\left\{V_{P D, i}\right\}$ after division by subsequently the transimpedance gain (leading to the photocurrent magnitudes $\left.\left\{I_{P D, i}\right\}\right)$ and the receiver responsivity [11]. 


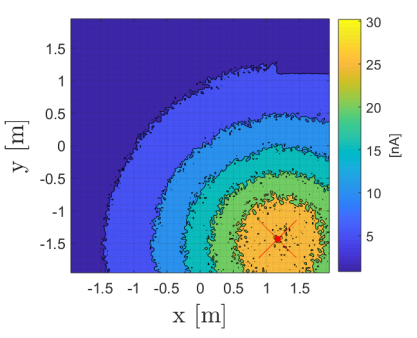

(a)

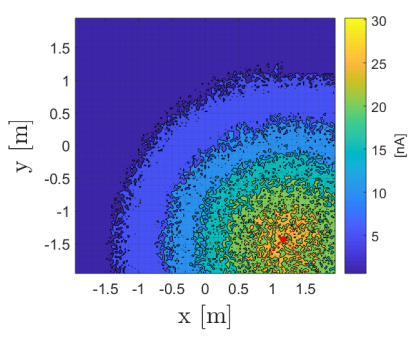

(b)

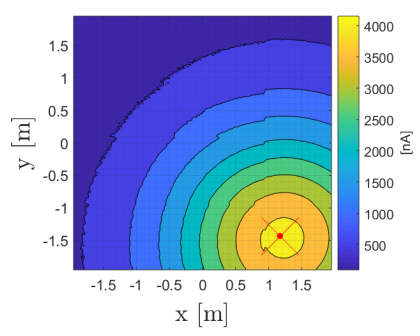

(c)

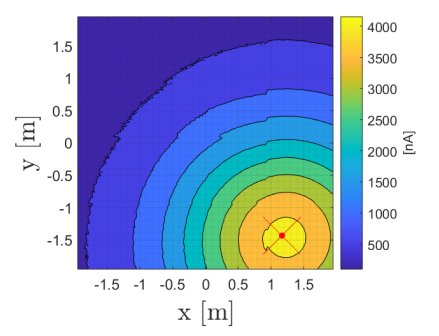

(d)

Fig. 2. Distribution of $I_{P D, 3}$ obtained via FFT-based demodulation with $A V G=16$ over $157^{2}$ grid points for uVLP (a) $P E A K$ and (b) $S P E C T$, and for VLP (c) $P E A K$ and (d) $S P E C T$. $2 \Delta f$ equals $10 \mathrm{~Hz}$ and $100 \mathrm{~Hz}$ for VLP and uVLP respectively. uVLP's $I_{P D, i}$ is similarly demodulated as VLP's.

2) Trilateration Positioning: Given the LEDs' coordinates $\left(x_{S, i}, y_{S, i}, z_{S, i}\right)$ and $\left\{P_{R, i}\right\}$, trilateration-based RSS first estimates the LED-PD distances $d_{i}$ by inverting a VLP channel model before computing a location estimate via least-squares minimisation of the linear system relating $d_{i}$ to $\left(x_{S, i}, y_{S, i}, z_{S, i}\right)$ (see [13] for details). The positioning performance is evaluated in terms of the root-mean-square error (rMSE), i.e. the Euclidean distance between the actual location and the estimated position.

3) Propagation Model: The employed VLP channel models are, as found in literature [14], the IR-propagation models of Kahn et al. [15]. The description of Kahn's models is the same as in previous work [11]. A cosine receiver angular acceptance is assumed.

4) Calibration: The LED coordinates $\left(x_{S, i}, y_{S, i}, z_{S, i}\right)$, $i=1 . . K$ equal $x_{S, i}=[-1.13,-1.12,1.17,1.15](\mathrm{m})$, $y_{S, i}=[-1.41,1.44,-1.43,1.4] \quad(\mathrm{m})$ and $z_{S, i}=$ $[2.26,2.26,2.25,2.26]$ (m).

Besides accurately knowing $\left(x_{S, i}, y_{S, i}, z_{S, i}\right)$, also $\left\{P_{t, i}\right\}$ and $\left\{f_{c, i}\right\}$ need to be a priori known. $\left\{f_{c, i}\right\}$ is determined with a single measurement per LED (see section II-B). $P_{t, i}$ generally is too [16]. However, a more robust method entails taking the mean of $P_{t, i} \cdot\left(d_{i} / z_{S, i}\right)^{3}$ for all grid points within a $10 \mathrm{~cm}$ radius of $\mathrm{LED}_{i}$ 's projection.

\section{EXPERIMENTAL Results}

\section{A. Measuring the Characteristic Frequency}

Fig. 3 depicts the MUltiple SIgnal Classification (MUSIC) [17] pseudospectrum of subspace order 20 of the total photovoltage time domain signal $V_{P D, i}(t)$ for each of the 4 COBs. The LEDs' $C F$ s are identified via peak detection to be $76.57 \mathrm{kHz}, 81.82 \mathrm{kHz}, 83.49 \mathrm{kHz}$ and $86.09 \mathrm{kHz}$. The minimal LED spread is $1.67 \mathrm{kHz}$, which in turn dictates a minimal $N$ (with $N$ the number of $V_{P D, i}(t)$ samples). These BXRE measurements allow concluding that the $C F$ is dependent on the individual LED lamp's characteristics, and that the $C F$ s are prominent enough to use as a basis for positioning. With the $C F \mathrm{~s}$ up to and exceeding $80 \mathrm{kHz}$, uVLP dictates a sufficiently high $f_{S}$ or necessitates the use of the $C F$ aliases. For the former, $f_{S}$ should exceed 2 times the maximum $C F$, provided the absence of higher frequency components that could alias into the $C F$ range.

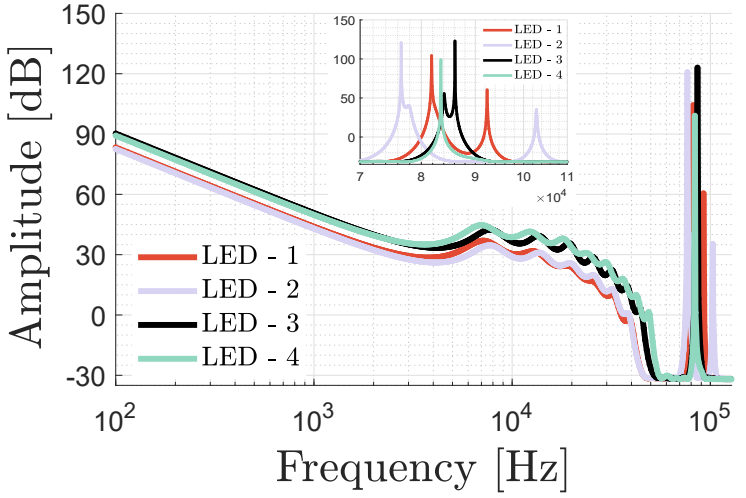

Fig. 3. MUSIC pseudospectrum versus frequency on a logarithmic scale of the 4 BXRE-35E2000-C-73 COB LEDs

\section{B. Measuring the $I_{P D, i}$ Propagation}

Given $f_{c, i}$, the photocurrent contributions $I_{P D, i}$ are demodulated with $A V G=2^{4}=16 . A V G=16$, also the FFT resolution in Hertz, is chosen as to discern between $f_{c, i}$ frequencies spread at least $16-20 \mathrm{~Hz}$ apart. A contour plot of the $I_{P D, i}$ distribution appertaining to LED 3 is shown in Fig. 2 for uVLP (a) $P E A K$ and (b) SPECT, and for VLP (c) $P E A K$ and (d) SPECT.

Fig. 2 (a) and (b) demonstrate that the uVLP PEAK $I_{P D, i}$ distribution exhibits less noisy, i.e. capricious behaviour than $S P E C T$ 's. This is due to the instability of the (instantaneous) $C F$. During VLP on the other hand, $P E A K$ and $S P E C T$ effectuate the same $I_{P D, i}$ as $f_{c, i}$ is stable. As $S P E C T$ is of lower complexity, it is the designated demodulation technique for VLP. Finally, comparing Fig. 2 (a)/(b) with (c)/(d) permits concluding that uVLP's $I_{P D, i}$ is 2 orders of magnitude smaller than VLP's.

As the variance on $I_{P D, i}$ is correlated with the localisation precision, it is interesting to plot the spatial distribution of the standard deviation on the VLP PEAK $I_{P D, 4}$ of LED 4 (in Fig. 4). This standard deviation is computed based on the $I_{P D, i}$ of each of the $A V G=16$ segments.

Fig. 4 shows that the standard deviation profile on $I_{P D, i}$ shows a significant contribution of the neighbouring (both in space and frequency) LEDs i.e. LED interference (mostly of LED 3 located at the bottom right). This extra contribution 


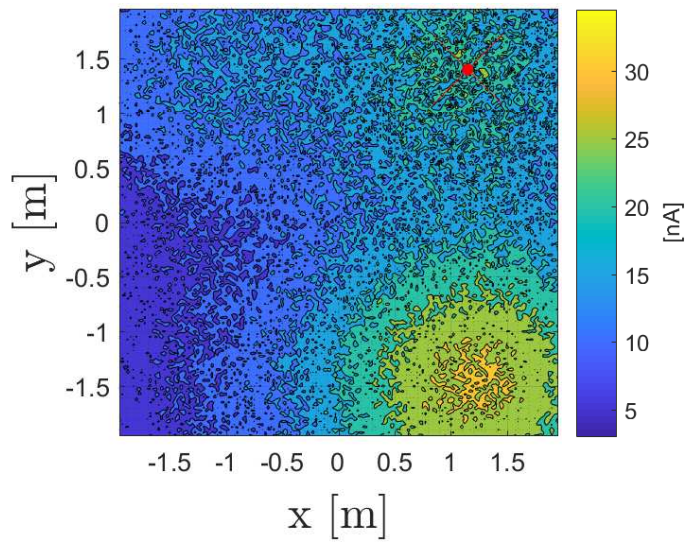

Fig. 4. Per grid point standard deviation of the $A V G=16$ segments' $I_{P D, 4}$ ( $I_{P D, i}$ for LED 4) measurement for VLP Peak

can be attributed to the real $f_{c, i}$ not being exact multiples of the ground frequency $f_{0}$. For uVLP, LED interference is not the dominant factor. There, the standard deviation has a strongly proportional component to the $I_{P D, i}$ magnitude. Also noticeable is that VLP's deviation's magnitude is two orders of magnitude smaller than the signal's. A final side note is that, for the setup under consideration, the deviation's amplitude is comparable to uVLP's $I_{P D, i}$.

\section{Positioning Results}

The cumulative distribution functions (CDFs) of the rMSE found during trilateration with the $(A V G=16) I_{P D, i} / P_{R, i}$ obtained in uVLP/VLP localisation with $P E A K / S P E C T$ demodulation, are shown in Fig. 5.

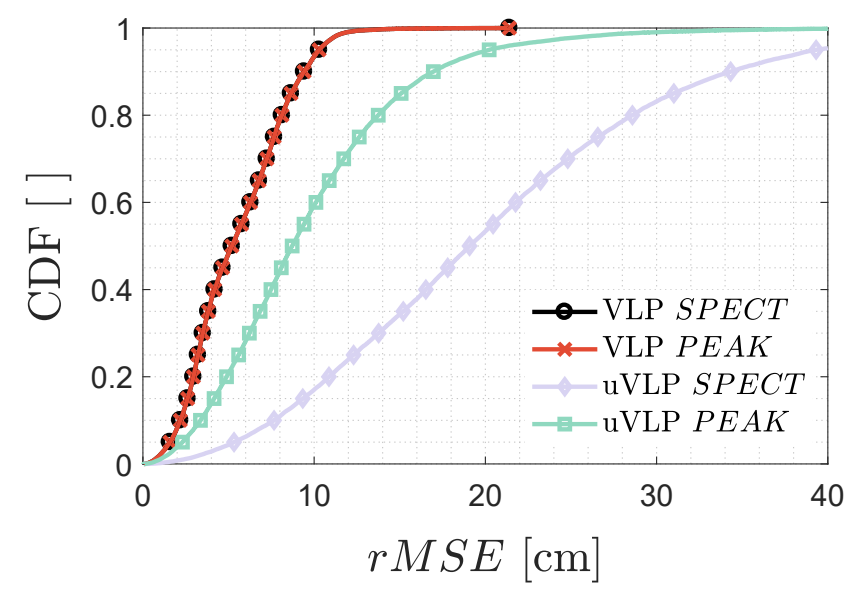

Fig. 5. CDF of the (u)VLP positioning rMSE with $P E A K(p)$ and $S P E C T$ (s) demodulation. The $P E A K$ and $S P E C T$ curve related to VLP overlap.

As expected (Section III-B), PEAK outscores $S P E C T$ when unmodulated LEDs are used. The $p_{50}, p_{75}$ and $p_{90}$ percentile rMSE improve respectively by $10.36 \mathrm{~cm}(54.3 \%)$, $13.94 \mathrm{~cm}(52.5 \%)$ and $17.4 \mathrm{~cm}(50.6 \%)$. The more than $50 \%$ postioning accuracy increase does come at the cost of additional complexity ( $A V G-1$ extra peak detects) and thus latency. The execution time rises 14 fold.

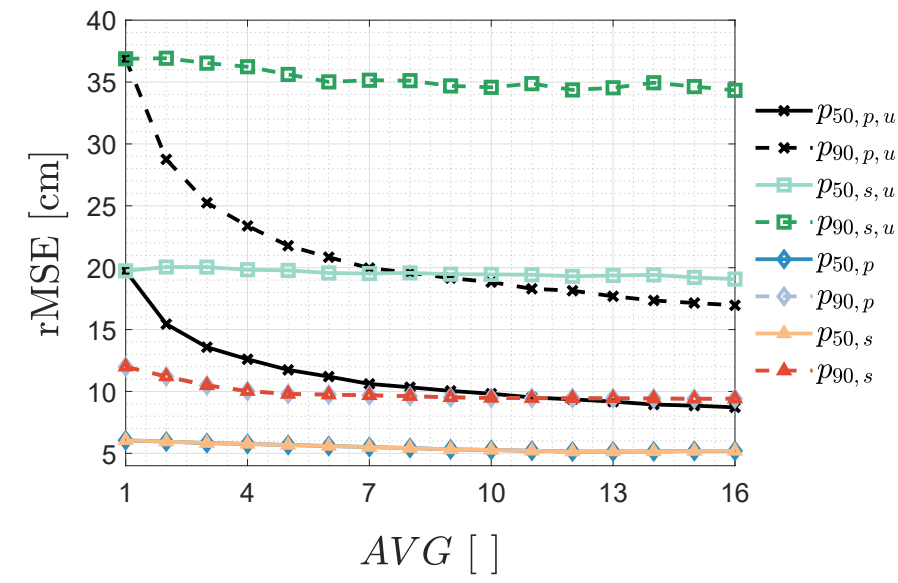

Fig. 6. Influence on the uVLP/VLP PEAK/SPECT $p_{50}$ and $p_{90}$ of averaging $A V G$ segments

Fig. 5 also demonstrates the limited loss of positioning accuracy of uVLP PEAK systems compared to their regular VLP counterparts. While VLP displays a $p_{50}=5.2 \mathrm{~cm}$, $p_{75}=7.67 \mathrm{~cm}$ and $p_{90}=9.42 \mathrm{~cm}$, uVLP only augments the rMSE to $8.72 \mathrm{~cm}, 12.63 \mathrm{~cm}$ and $16.96 \mathrm{~cm}$, respectively. Those values represent percentage increases of $67.8 \%, 64.7 \%$ and $80.1 \%$.

1) Positioning precision and accuracy: Main accuracy decreasing factors are identified to be LED interference (certainly for VLP, see section III-B), the LED tilt present (i.e. due to the nonideal lab setup) [11], the cosine normalised responsivity assumed [11], and measurement errors.

To estimate the portion of the positioning error that can be attributed to the noise influence, i.e. the localisation precision, the $A V G=16 I_{P D, i}$ values are reverted to. Per grid point, the standard deviation on both the localisation rMSE and the position estimate (i.e. Euclidean distance) of each segment, with respect to the mean $I_{P D, i}$ 's rMSE and location, is computed. The $50^{\text {th }}$ and $90^{\text {th }}$ percentile deviation on the localisation error and the positioning estimate are denoted by $p_{50, \text { err }} / p_{90, \text { err }}$ and $p_{50, \text { dist }} / p_{90, \text { dist }}$.

For uVLP, $p_{50, e r r}$ and $p_{90, e r r}$ equal $15.6 \mathrm{~cm}$ and $21.06 \mathrm{~cm}$, while $p_{50, \text { dist }}$ and $p_{90, \text { dist }}$ amount to $21.09 \mathrm{~cm}$ and $26.73 \mathrm{~cm}$. For VLP, these values are $2.2 \mathrm{~cm}, 5.75 \mathrm{~cm}, 3.26 \mathrm{~cm}$ and $8.5 \mathrm{~cm}$. However, the results of Fig. 5 are obtained with the averaged $I_{P D, i}$. Under assumptions (such as uncorrelatedness), the above numbers are reduced by a factor $\sqrt{A V G}$. From this approximate precision analysis, it can be concluded that a significant part of uVLP's loss of accuracy (compared to VLP) is due to its significantly lower signal-to-noise ratio (SNR). This loss of accuracy can be partly mitigated by averaging multiple $I_{P D, i}$ observations, at least as many as latency constraints allow. VLP's performance is mainly limited by consistent and bias inaccuracies, such as those listed in the beginning of this section.

2) Positioning rMSE as a function of $A V G$ : Fig. 6 depicts the $p_{50}$ and $p_{90}$ positioning errors for the different (de)modulation combinations as a function of the number 
TABLE I

COMPARISON OF UVLP AND 'REGULAR' VLP

\begin{tabular}{|l|l|l|}
\hline Description & VLP & uVLP \\
\hline Principle & & \\
Typical frequency range & up to MHz & $30-90 \mathrm{kHz}$ \\
Modulation Index & $50 \%$ & $0 \%$ \\
Average LED current & $50 \%$ & $100 \%$ \\
\hline Accuracy & & \\
Experimental $p_{50}$ & $5.20 \mathrm{~cm}$ & $8.72 \mathrm{~cm}$ \\
\hline Cost & LED driver & None \\
Retrofitting effort & Additional lamps & None \\
New infrastructure & Same & Same \\
Receiver-side Cost & \multicolumn{2}{|l}{} \\
\hline
\end{tabular}

of $(A V G)$ segments' $I_{P D, i}$ is averaged for positioning. The segment length remains constant, while the number of averaged $I_{P D, i}$ samples increases from 1 to 16. Fig. 6 again shows the superior performance of $P E A K$ over SPECT in uVLP. The latter being plagued by the $f_{c, i}$ time variation mismatch. At $A V G=16$, uVLP's $p_{50}$ and $p_{90}$ have not yet converged, meaning that uVLP's performance can still be ameliorated by increasing $A V G$. However, as the associated positioning update rates drop below $1 \mathrm{~Hz}$, such systems are deemed impractical for most real-time applications. A use case is still found in the tracking of slow-moving objects (and in non-real-time situations).

For VLP, $A V G=5$ seems to be the convergence start point. $p_{50}$ and $p_{90}$ only decrease by approximately $9.2 \%$ and $4.02 \%$ when averaging $I_{P D, i} 11$ times more. Stopping at $A V G=5$, allows to increase the update rate (more than) threefold.

3) Discussion of Results: Table I lists the principles and differences of both uVLP and regular VLP. FFT-based RSS uVLP exhibits $p_{90}$ positioning errors no longer contained in the subdecimetre range, effectively ruling uVLP systems out for highly-accurate applications such as the tracking of indoor unmanned robots. However, an accuracy below $30 \mathrm{~cm}$ does suffice for many other (broadcasting) applications. Certainly, as due to uVLP not requiring a dedicated infrastructure, lighting that is furthermore omnipresent, the roll-out costs are minimal. Only the tracked object needs to be equipped with a VLP-enabled receiver, which in itself is not of large cost.

\section{CONClusion And Future Work}

In this manuscript, it is shown that COB LEDs exhibit a characteristic frequency $(C F)$, which helps identifying and demultiplexing the LEDs' individual photovoltage contributions to be used in photodiode (PD)-based received signal strength (RSS) uVLP. In combination with trilateration and FFT-based demodulation in a $4 \mathrm{~m}$ by $4 \mathrm{~m}$ lab setup, uVLP increases respectively the $p_{50}$ and $p_{90}$ rMSE from $5.2 \mathrm{~cm}$ to $8.72 \mathrm{~cm}$ and from $9.42 \mathrm{~cm}$ to $16.96 \mathrm{~cm}$ compared with VLP.
This manuscript shows uVLP's potential for many localisation applications such as tracking hospital beds, office room laptops (equipped with a PD), virtual and augmented reality.

Boasting the uVLP positioning performance via improved demodulation and positioning methods for both point sourcelike and non-point source LEDs encompasses the future work. uVLP's aptitude in industrial settings should also be examined.

\section{REFERENCES}

[1] 5G PPP, "Internet of Radio Light (IORL)," https://iorl.5g-ppp.eu/ (accessed May 1, 2020).

[2] J. Cosmas, Y. Zhang and X. Zhang, "Internet of Radio-Light: 5G Broadband in Buildings," 23th European Wireless 2017 Conference, Dresden, Germany, 2017, pp. 74-79.

[3] J. Cosmas et al., "A Scalable and License Free 5G Internet of Radio Light Architecture for Services in Homes \& Businesses," IEEE International Symposium on Broadband Multimedia Systems and Broadcasting (BMSB), Valencia, Spain, 2018, pp. 1-6.

[4] J. Cosmas et al., "5G Internet of radio light services for Musée de la Carte Jouer," Global LIFI Congress (GLC), Paris, France, 2018, pp. $1-6$.

[5] J. Cosmas et al., "5G Internet of radio light services for supermarkets," 14th China International Forum on Solid State Lighting: International Forum on Wide Bandgap Semiconductors China (SSLChina: IFWS), Beijing, China, 2017, pp. 69-73.

[6] L. Shi et al., "5G Internet of Radio Light Positioning System for Indoor Broadcasting Service," in IEEE Transactions On Broadcasting, 2020.

[7] B. Meunier and J. Cosmas, "5G Internet of Radio Light Virtual Reality System," IEEE International Symposium on Broadband Multimedia Systems and Broadcasting (BMSB), Valencia, Spain, 2018, pp. 1-5.

[8] R. Amsters, E. Demeester, P. Slaets and N. Stevens, "Unmodulated Visible Light Positioning Using the Iterated Extended Kalman Filter," 2018 International Conference on Indoor Positioning and Indoor Navigation (IPIN), Nantes, France, 2018, pp. 1-8.

[9] C. Zhang and X. Zhang, "Visible Light Localization Using Conventional Light Fixtures and Smartphones," in IEEE Transactions on Mobile Computing, vol. 18, no. 12, pp. 2968-2983, 2019.

[10] H. van der Broeck, G. Sauerlander and M. Wendt, "Power driver topologies and control schemes for LEDs," Twenty-Second Annual IEEE Applied Power Electronics Conference and Exposition (APEC 07), Anaheim, USA, 2007, pp. 1319-1325.

[11] S. Bastiaens, W. Raes, N. Stevens, L. Martens, W. Joseph and D. Plets, "Impact of a Photodiode's Angular Characteristics on RSS-Based VLP Accuracy," in IEEE Access, vol. 8, pp. 83116-83130, 2020.

[12] S. De Lausnay, L. De Strycker, J. P. Goemaere, N. Stevens and B. Nauwelaers, "A Visible Light Positioning system using Frequency Division Multiple Access with square waves," International Conference on Signal Processing and Communication Systems, Cairns, Australia, 2015, pp. 1-7.

[13] W. Gu, M. Aminikashani, P. Deng and M. Kavehrad, "Impact of Multipath Reflections on the Performance of Indoor Visible Light Positioning Systems," Journal of Lightwave Technology, vol. 34, pp. 2578-2587, 2016.

[14] Y. Zhuang et al., "A Survey of Positioning Systems Using Visible LED Lights," in IEEE Communications Surveys \& Tutorials, vol. 20, no. 3, pp. 1963-1988, 2018.

[15] J. M. Kahn and J. R. Barry, "Wireless infrared communications," in Proceedings of the IEEE, vol. 85, no. 2, pp. 265-298, 1997.

[16] D. Plets, S. Bastiaens, L. Martens, W. Joseph and N. Stevens, "On the impact of LED power uncertainty on the accuracy of 2D and 3D visible light positioning," in OPTIK, vol. 195, pp. 1-12, 2019.

[17] R. Schmidt, "Multiple Emitter Location and Signal Parameter Estimation," in IEEE Transactions on Antennas and Propagation, vol. 34, no. 3, pp. 276-280, 1986. 